\title{
Psychological factors involved in auditory presence
}

\author{
Kenji Ozawa ${ }^{1}$, Yoshihiro Chujo ${ }^{1}$, Yôiti Suzuki ${ }^{2}$ and Toshio Sone ${ }^{3}$ \\ ${ }^{1}$ Faculty of Engineering, Yamanashi University, \\ 4-3-11 Takeda, Kofu, 400-8511 Japan \\ ${ }^{2}$ Research Institute of Electrical Communication/Graduate School of Information Sciences, Tohoku University, \\ 2-1-1 Katahira, Aoba-ku, Sendai, 980-8577 Japan \\ ${ }^{3}$ Faculty of Systems Science and Technology, Akita Prefectural University, \\ 84-4 Ebinokuchi, Tsuchiya, Honjo, 015-0055 Japan
}

(Received 9 July 2002, Accepted for publication 1 August 2002)

Keywords: Auditory presence, Binaural reproduction, SD method, Factor analysis PACS number: 43.66.Lj, 43.66.Pn

\section{Introduction}

The authors are conducting a series of studies on the quantitative description of impression called "rinjôkan" in Japanese [1]. This word may be translated as "presence" [2] with the meaning that one has the illusion of being in some place or environment, even when one is physically situated in another. In this report, "presence" is used in this way.

This impression seems to be a principal factor in the evaluation of the performance of present AV (Audio-Visual) media. However, the characteristics of presence have not been sufficiently clarified. Presence may be a multimodal impression, and thus the authors address auditory presence as the first step to obtain a full understanding of the characteristics of this impression [1]. In this report, the relation between auditory presence and psychological factors of sound perception, i.e., factors of sound quality, information on sound, and sound localization [3-5], is investigated.

\section{Psychoacoustical experiment}

\subsection{Stimuli}

Fourteen sounds with a duration of $20 \mathrm{~s}$ were binaurally recorded using a dummy head (Koken, Samurai). They are classified into three groups as shown in Table 1 with respect to the location of sound sources relative to a listener as follows:

Group I: Sound sources were moving.

Group II: Sound sources were moving with the listener.

Group III: Sound sources were stationary.

The sounds were presented to a subject via headphones (Stax, SR-Lambda). As shown in Table 1, the number of stimuli was twenty-four since some of the fourteen sounds were reproduced by the following three methods:

Method A: The frequency-response characteristics of the outer ears of the dummy head were rectified through digital signal processing prior to the reproduction to avoid the duplication of the characteristics in recording and reproduction [3].

Method B: Besides method A, cross talk of $-6 \mathrm{~dB}$ was supplied between the two channels of the binaural sound. The overall level was maintained so as to be the same as that of the sound in method A. This method was applied to simulate a stereophonic reproduction system with two loudspeakers.

Method C: Besides method A, diotic sound was synthesized by summing up the two-channel signals of the binaural sound. The overall level was maintained in the same way as in method B. This method was applied to simulate a monophonic system.

\subsection{Experimental procedure}

An experiment was carried out by the SD (Semantic Differential) method. Four male subjects, between 21 and 23 years of age, participated in the experiment. Each stimulus was presented repeatedly to the subjects until their judgments were over. The subjects rated their impressions of the stimulus on a seven-point category scale for thirty-four pairs of adjectives. As shown in Table 2, the pairs of adjectives were taken from previous studies in which sound perception was investigated [3-5]. The pair of "having high presence-having low presence" was included in order to evaluate the auditory presence directly.

\section{Factor analysis}

The data for thirty-three pairs of adjectives, excluding that for the pair of the auditory presence, were analyzed with the method of factor analysis. Figure 1 shows the eigenvalues derived from the analysis. If the criterion that eigenvalues are more than 1 were applied, six factors would be extracted. However, since the extracted 6th factor was difficult to explain and the difference of eigenvalues between the 5th and 6th factors was large, five factors were further considered.

The factor loadings of the pairs of adjectives for the five factors are shown in Table 2, in which the pairs are sorted by the largest factor loading for each pair shown by underline. The first factor, F1, is regarded to represent "aesthetic state" because the pairs such as "beautiful-dirty" and "comfortable-uncomfortable" have the largest loadings on this factor. Similarly, F2 to F5 are regarded to represent "volume," "softness," "information on sound," and "sound localization," respectively. These factors show good agreement with the psychological factors of sound perception shown in the literatures [3-5]. 
Table 1 Stimuli classified into three groups: groups I, II, and III. Some of the sounds were reproduced by reproduction methods $\mathrm{A}, \mathrm{B}$, and $\mathrm{C}$ (See the text for details).

\begin{tabular}{|c|c|}
\hline & Primary sound involved in the stimuli \\
\hline I & $\begin{array}{l}\text { sound of a train passing (A, B, C) } \\
\text { another person's footsteps on fallen leaves }(\mathrm{A}, \mathrm{B}, \mathrm{C}) \\
\text { sound of vehicles passing in a tunnel (A) } \\
\text { sound heard while riding a bicycle (A) } \\
\text { sound of vehicles passing on a road (A) } \\
\text { sound heard in a moving car (A) }\end{array}$ \\
\hline II & $\begin{array}{l}\text { the listener's footsteps on fallen leaves (A, B, C) } \\
\text { the listener's footsteps in a corridor (A) }\end{array}$ \\
\hline III & $\begin{array}{l}\text { hum of voices in a concert hall }(\mathrm{A}, \mathrm{B}, \mathrm{C}) \\
\text { music reproduced at the side of a PC (A, B, C) } \\
\text { murmuring of a stream (A) } \\
\text { piano music in a concert hall (A) } \\
\text { sound at a railroad crossing (A) } \\
\text { hum of voices at a public square (A) }\end{array}$ \\
\hline
\end{tabular}

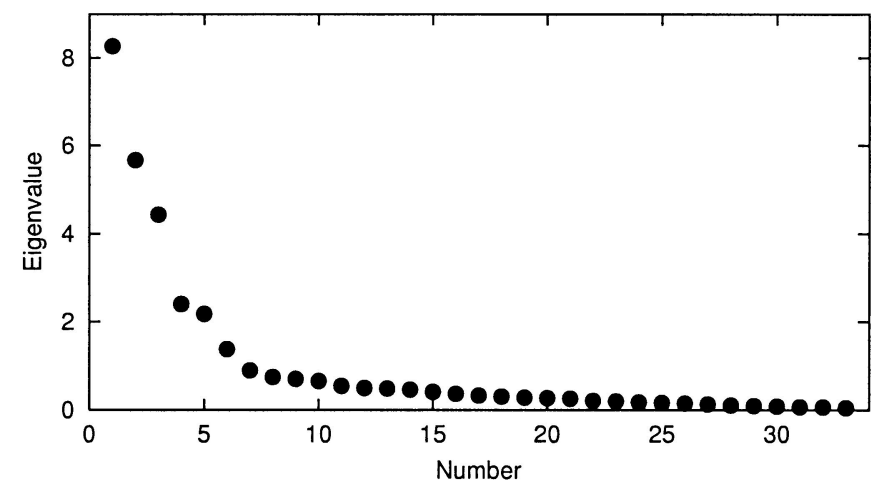

Fig. 1 Eigenvalues obtained from the factor analysis.

Table 2 Factor loadings for thirty-three pairs of adjectives after Varimax rotation. The largest factor loading for each pair is underlined. The definitions of numbers $1-4$ are given below the table.

\begin{tabular}{|c|c|c|c|c|c|}
\hline Pair of adjectives & $\mathrm{F} 1$ & $\mathrm{~F} 2$ & $\mathrm{~F} 3$ & $\mathrm{~F} 4$ & F5 \\
\hline good-bad & $\underline{0.85}$ & -0.06 & 0.04 & 0.20 & 0.14 \\
\hline willing to stay-not willing to stay & $\underline{0.84}$ & -0.09 & -0.01 & -0.05 & 0.19 \\
\hline comfortable-uncomfortable & $\overline{0.82}$ & -0.08 & -0.01 & 0.00 & 0.01 \\
\hline tranquilizing-irritating & $\overline{0.81}$ & -0.34 & -0.11 & -0.06 & 0.10 \\
\hline beautiful-dirty & $\overline{0.78}$ & -0.20 & -0.06 & 0.06 & -0.15 \\
\hline interesting-boring & $\overline{0.73}$ & 0.41 & 0.00 & -0.07 & 0.00 \\
\hline foul-pure & $-\overline{0.61}$ & 0.46 & -0.03 & -0.24 & 0.09 \\
\hline rough-smooth & -0.67 & 0.33 & 0.09 & -0.33 & 0.04 \\
\hline informative-empty & -0.10 & $\underline{0.83}$ & -0.10 & -0.12 & 0.04 \\
\hline loud-soft & -0.14 & $\underline{0.79}$ & 0.18 & 0.03 & 0.01 \\
\hline strong-weak & -0.13 & 0.78 & 0.26 & 0.03 & 0.06 \\
\hline powerful-powerless & -0.14 & $\overline{0.78}$ & 0.15 & 0.15 & 0.09 \\
\hline noisy-calm & -0.29 & $\overline{0.77}$ & 0.12 & -0.11 & -0.15 \\
\hline rich-poor & 0.24 & $\overline{0.76}$ & -0.16 & 0.06 & -0.09 \\
\hline speedy-slow & -0.02 & $\overline{0.65}$ & 0.50 & 0.21 & 0.10 \\
\hline dangerous-safe & -0.40 & 0.51 & 0.48 & 0.18 & 0.17 \\
\hline simple-complex & 0.14 & $-\overline{0.85}$ & 0.16 & 0.11 & 0.09 \\
\hline sharp-dull & 0.03 & 0.34 & 0.74 & 0.05 & 0.00 \\
\hline clear-dim & 0.32 & -0.02 & $\underline{0.68}$ & 0.23 & -0.24 \\
\hline crisp-ambiguous & 0.44 & -0.23 & $\overline{0.60}$ & 0.12 & -0.26 \\
\hline metallic-deep & -0.04 & 0.35 & $\underline{0.58}$ & 0.03 & 0.16 \\
\hline cold-warm & -0.45 & -0.23 & $\underline{0.56}$ & 0.19 & 0.28 \\
\hline soft-hard & 0.29 & 0.06 & $-\overline{0.73}$ & 0.14 & -0.21 \\
\hline natural-unnatural & 0.00 & -0.13 & -0.10 & $\underline{0.83}$ & 0.00 \\
\hline$\left({ }^{*} 1\right)$ & 0.05 & 0.08 & 0.04 & $\underline{0.82}$ & 0.19 \\
\hline familiar-unfamiliar & -0.10 & 0.14 & 0.16 & $\underline{0.71}$ & -0.10 \\
\hline$(* 2)$ & 0.46 & -0.10 & 0.20 & $\underline{0.68}$ & -0.08 \\
\hline$(* 3)$ & 0.21 & -0.01 & 0.18 & 0.59 & 0.66 \\
\hline$(* 4)$ & 0.24 & -0.04 & 0.18 & 0.52 & $\overline{0.63}$ \\
\hline dynamic-static & -0.28 & 0.23 & 0.40 & 0.11 & $\overline{0.53}$ \\
\hline dark-light & -0.44 & -0.36 & 0.39 & -0.06 & 0.52 \\
\hline extensive-not extensive & 0.36 & 0.26 & -0.09 & 0.31 & $\overline{0.52}$ \\
\hline high-low & -0.01 & 0.11 & 0.11 & 0.27 & $-\overline{0.76}$ \\
\hline
\end{tabular}

*1: easy to image the scene-difficult to image the scene

*2: easy to understand the sound source-difficult to understand the sound source

*3: easy to perceive the distance to the sound source-difficult to perceive the distance to the sound source

*4: easy to perceive the direction of the sound source-difficult to perceive the direction of the sound source 


\section{Multiple regression analysis for auditory presence}

In order to examine the relation between the auditory presence and the factors of sound perception, multiple regression analysis was carried out. In the analysis, the presence of each stimulus was a criterion variable, and the five factor scores of the stimulus were explanatory variables. As a result, the multiple correlation coefficient was 0.95 . This indicates that the auditory presence can be considered to be expressed by a linear combination of the factors of sound perception. The partial regression coefficients for the factor scores of F1 to F5 were $0.51,0.30,0.24,0.87$, and 0.87 , respectively. The larger coefficients for F4 and F5 among the five factors imply that the principal factors of auditory presence are "information on sound," and "sound localization." This suggests that the following two points are important to obtain high auditory presence: One is that sufficient information on sound should be given so that a listener can image the original scene naturally. The other is that sound sources should be well localized.

\section{Conclusion}

Auditory presence is shown to be well expressed by a linear combination of the factors of sound perception, which have been obtained in previous investigations [3-5]. Further study should examine the effect of visual information on auditory presence to further clarify the multimodal nature of the impression of presence.

\section{Acknowledgements}

A part of this study was supported by the Sound Technology Promotion Foundation in FY 1998 and the Ministry of Education, Science, Sports and Culture in FYs 1999 and 2000 (No. 11832008). This research was conducted under the Cooperative Research Project Program of the Research Institute of Electrical Communication, Tohoku University (No. H12/A05).

\section{References}

[1] K. Ozawa, Y. Chujo, Y. Suzuki and T. Sone, "Effects of contents and methods of reproduction on the auditory presence," Proc. Autumn Meet. Acoust. Soc. Jpn., pp. 611612 (1999).

[2] R. M. V. Collik, K. Hinata and M. Tanabe, Eds., Kenkyusha's New College Japanese-English Dictionary (Kenkyusha, Tokyo, 1995), p. 1966.

[3] K. Abe, K. Ozawa, Y. Suzuki and T. Sone, "Evaluation of environmental sounds using adjectives describing sound quality, emotional state, and information carried by sounds," J. Acoust. Soc. Jpn. (J), 54, 343-350 (1998).

[4] T. Sone, K. Kido and T. Nimura, "Factor analysis of descriptive adjectives for the evaluation of sounds," J. Acoust. Soc. Jpn. (J), 18, 320-326 (1962).

[5] O. Kitamura, S. Namba and R. Matsumono, "Factor analytical research of tone color," Proc. 6th Int. Congr. on Acoust., A-511 (1968). 